\title{
Illustrated catalogue of North American species of Ctenopbion Horstmann and Pbradis Förster (Hymenoptera: Ichneumonidae: Tersilochinae)
}

\section{ИАлюстрированный каталог североамериканских видов Ctenopbion Horstmann u Pbradis Förster (Hymenoptera: Ichneumonidae: Tersilochinae)}

\author{
A.I. Khalaim
}

А.И. Халаим

Universidad Autünoma de Tamaulipas, Ciudad Victoria, Tamaulipas, Mexico.

Zoological Institute of the Russian Academy of Sciences, St. Petersburg, Universitetskaya nab. 1, Russia. E-mail: ptera@mail.ru Зоологический институт РАН, Санкт-Петербург, Университесткая наб. 1, Россия.

KEY WORDS: Nearctic region, North America, catalogue, type, Ctenophion, Phradis, parasitoids.

КЛЮЧЕВЫЕ СЛОВА: Неарктика, Северная Америка, каталог, тип, Ctenophion, Phradis, паразитоиды.

ABSTRACT. Illustrated catalogue of North American species of genera Ctenophion Horstmann, 2010 (1 species) and Phradis Förster, 1869 (20 species) is provided. Colour photographs of holotypes of 18 species of Phradis are given for the first time.

РЕЗЮМЕ. Дан иллюстрированный каталог североамериканских видов родов Ctenophion Horstmann, 2010 (1 вид) и Phradis Förster, 1868 (20 видов). Цветные фотографии голотипов 18 видов Phradis публикуются впервые.

\section{Introduction}

First North American species of the genus Phradis Förster, 1869, P. kasparyani Khalaim, 2002, was described by Khalaim [2002] from California. Horstmann [2013] revised the Nearctic fauna of the genus, described 17 new species from Canada and USA, and provided an identification key to 18 Nearctic species. Recently, two rare species, Phradis bufalosus Khalaim et Ruíz-Cancino, 2018 and $P$. nanacamilpus Khalaim et Ruíz-Cancino, 2018, were discovered from Central Mexico [Khalaim, Ruíz-Cancino, 2018]. Thus, the North American fauna of the genus currently comprises 20 known species; of them 18 species described before 2018 were illustrated only by scarce contour figures. The genus Ctenophion Horstmann, 2010 was described for one species, C. niger Horstmann, 2010, which was also scarcely illustrated only by contour figures.
The aim of this work is to give a catalogue of Nearctic species of Ctenophion and Phradis with data on holotype and general distribution to each species, and provide high quality colour photographs taken primarily from holotypes.

\section{Material and Methods}

Following acronyms of depositories are used in this work: AEIC - Townes et Dasch collections (former American Entomological Institute), recently moved to the Utah State University, Logan, Utah, USA; CNC Canadian National Collection of Insects, Ottawa, Ontario, Canada; ESUW - University of Wyoming, Laramie, Wyoming, USA; TAMU — Texas A\&M University, College Station, Texas, USA; UNAM — Instituto de Biología, Universidad Nacional Autónoma de México, D.F., Mexico; ZIN — Zoological Institute RAS, St Petersburg, Russia; ZSM — Zoologische Staatssammlung, Munich, Germany.

Paratype of $C$. niger and holotypes of 17 species of Phradis were examined and photographed using a digital camera Nikon 1 V3 + Leica Z16 APO + Leica Planaro 1.0x / WD $97 \mathrm{~mm}$ during my visit to the ZSM in May 2016; these specimens (except paratype of $C$. niger belonging to ZSM) are temporary stored in the ZSM and will be returned to USA and Canadian collections. Photographs of P. kasparyani (Figs 35-40) were taken in the ZIN with a Canon EOS 70D digital camera attached to an Olympus SZX10 stereomicroscope, and partially focused images were combined using Helicon

How to cite this article: Khalaim A.I. 2019. Illustrated catalogue of North American species of Ctenophion Horstmann and Phradis Förster (Hymenoptera: Ichneumonidae: Tersilochinae) // Russian Entomol. J. Vol.28. No.4. P.400-420. doi: 10.15298/rusentj.28.4.09 
Focus Pro software. Map of North America with type localities (Fig. 1) was generated from the Google Maps service (https://www.google.com/maps/); supplementary kmz-file containing detailed georgaphic data is enclosed.

Countries and regions in Distribution sections are listed in alphabetical order. Non-type males and specimens of Phradis mentioned by Horstmann [2010] in Variation sections are included to general distribution as doubtful (with a question mark “?”).

\section{Results}

Illustrated catalogue of the Nearctic species of the genera Ctenophion (one species) and Phradis (20 species) is given. Colour photographs are provided for the single species of Ctenophion and 18 species of Phradis; photographs of two species, $P$. bufalosus and $P$. nanacamilpus, recently described from Mexico are not included because these two species were well illustrated in the original publication [Khalaim, Ruíz-Cancino,

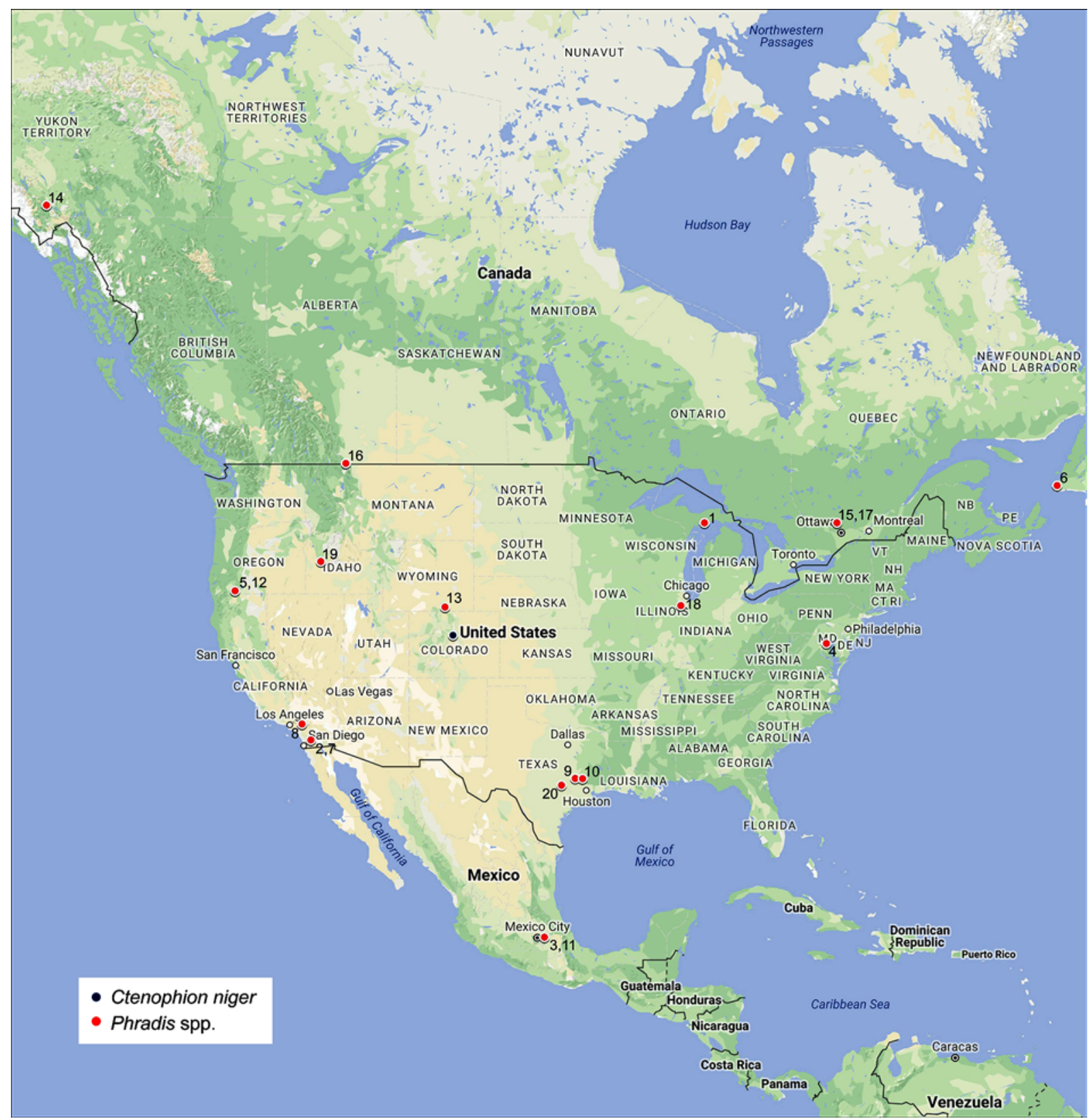

Figs 1. Map of North America with type localities of Ctenophion and Phradis species. Numbers of localities in the map correspond with those of Phradis species in the catalogue.

Рис. 1. Карта Северной Америки с типовыми локациями видов Ctenophion и Phradis. Номера точек на карте соответствуют номерам видов Phradis в каталоге. 
2018]. Type localities of Ctenophion niger and 20 species of Phradis are presented in the map (Fig. 1); see supplementary kmz-file for detailed geographic data.

Genus Ctenophion Horstmann, 2010

Type species: Ctenophion niger Horstmann, 2010.

DESCRIPTION. Relatively large species with body length $4.0-5.5 \mathrm{~mm}$. Head, in dorsal view, with gena roundly tapered behind eyes, almost as long as eye width. Antennal flagellum long, filiform, with 24-25 flagellomeres; several subbassal flagellomeres bear distinct finger-shaped structures on outer side near apex. Mandible with upper tooth about as long as lower tooth. Malar space longer than basal mandibular width. Clypeus broad, lenticular. Hypostomal carina obliterated.

Notaulus distinct, with irregular wrinkles. Epicnemial carina with upper end bent forwards and reaching front

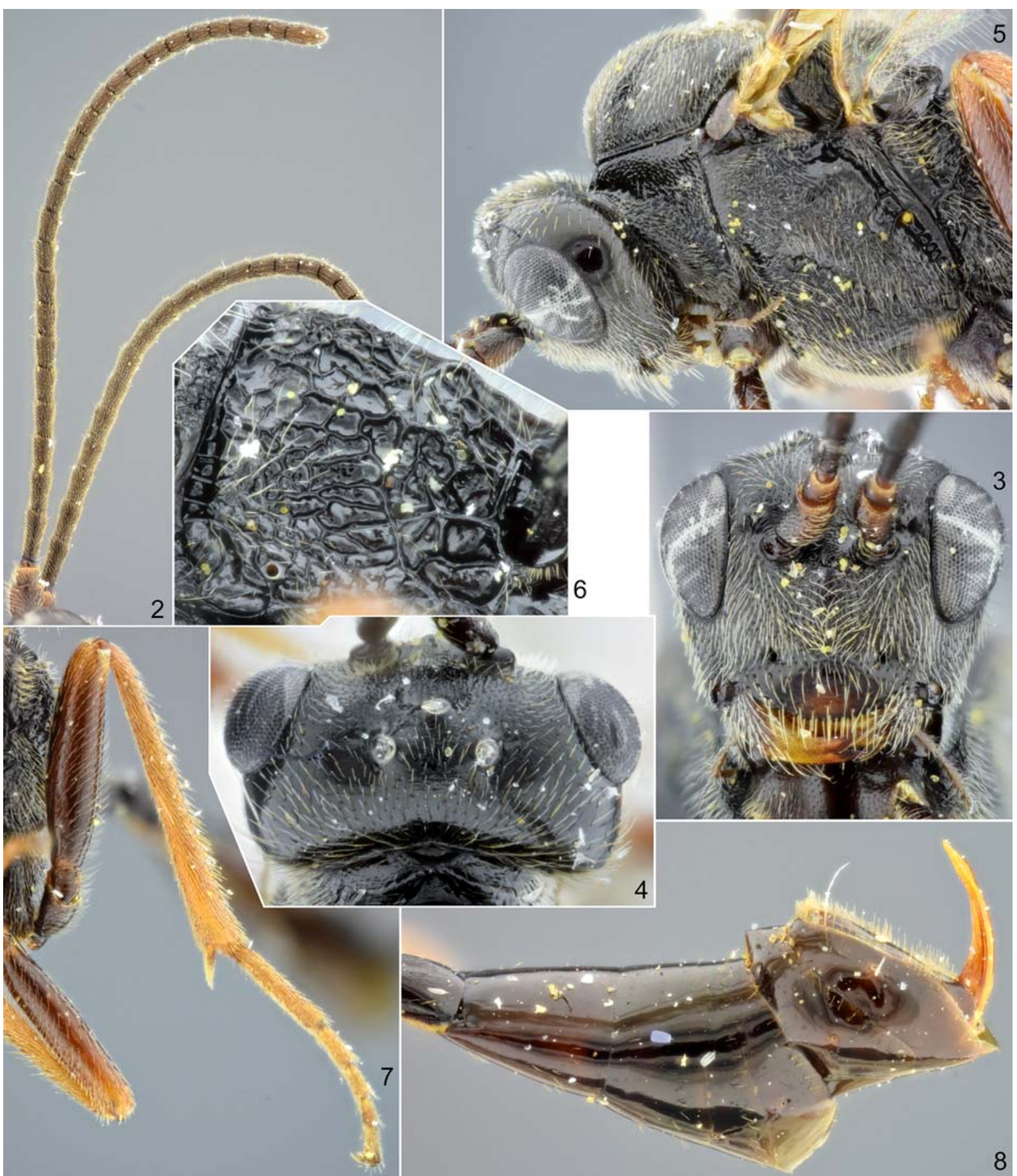

Figs 2-8. Ctenophion niger, paratype female: 2 - antennae, lateral view; 3 - head, front view; 4 - head, dorsal view; 5 - head and mesosoma, lateral view; 6 - propodeum, dorsolateral view; 7 - hind leg, lateral view; 8 - apex of metasoma with ovipositor, lateral view.

Рис. 2-8. Ctenophion niger, паратип, самка: 2 - антенны, сбоку; 3 - голова, спереди; 4 - голова, сверху; 5 - голова и мезосома, сбоку; 6 - проподеум, сверху и сбоку; 7 - задняя нога, сбоку; 8 - вершина метасомы с яйцекладом, сбоку. 
margin of mesopleuron slightly below middle of posterior margin of pronotum. Foveate groove broad and coarse, somewhat upcurved anteriorly, extending from epicnemial carina to base of mid coxa. Propodeum with coarse irregular rugae (Fig. 6). Propodeal spiracle round, separated from pleural carina by about 3.0 times its own diameter.

Fore wing with second recurrent vein $(2 \mathrm{~m}-\mathrm{cu})$ interstitial, intercubitus (2rs-m) unusually long. First and second sections of radius (Rs+2r and Rs) meeting at obtuse angle. Second abscissa of postnervulus present, thus brachial cell is closed posteriorly. Hind wing with nervellus (cu1\&cu-a) nearly vertical.
Legs slender. Hind leg with tibia much longer than femur; basitarsus short, about 0.4 times as long as tibia (Fig. 7). Longer hind tibial spur almost straight, with distal end slightly curved. Tarsal claws distinctly pectinate.

Metasomal tergite 1 robust, depressed; glymma deep, situated in centre of first tergite, joining by furrow with ventral part of postpetiole; petiole entirely striate laterally before glymma. Ovipositor very short and robust, upcurved, with shallow dorsal subapical depression (Fig. 8).

COMPOSITION. One species described from the Nearctic region and recently recorded from North Europe [Khalaim, Várkonyi, 2018].

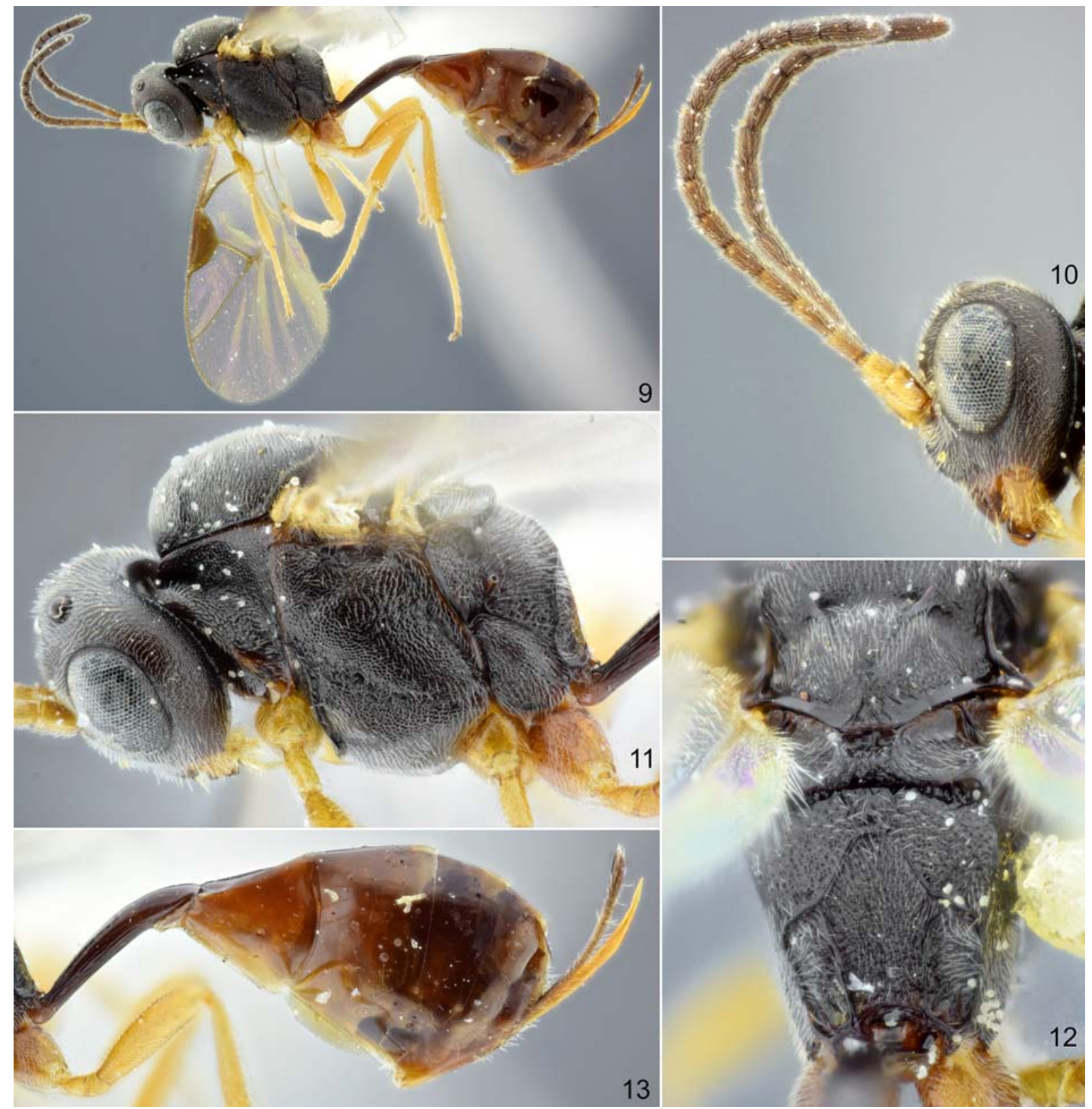

Figs 9-13. Phradis basalis, holotype female: 9 - habitus, lateral view; 10 - head with antennae, lateral view; 11 — head and mesosoma, lateral view; 12 - propodeum, dorsal view; 13 - metasoma with ovipositor, lateral view.

Рис. 9-13. Phradis basalis, голотип, самка: 9 — габитус, сбоку; 10 — голова с антеннами, сбоку; 11 — голова и мезосома, сбоку; 12 - проподеум, сверху; 13 - метасома с яйцекладом, сбоку. 


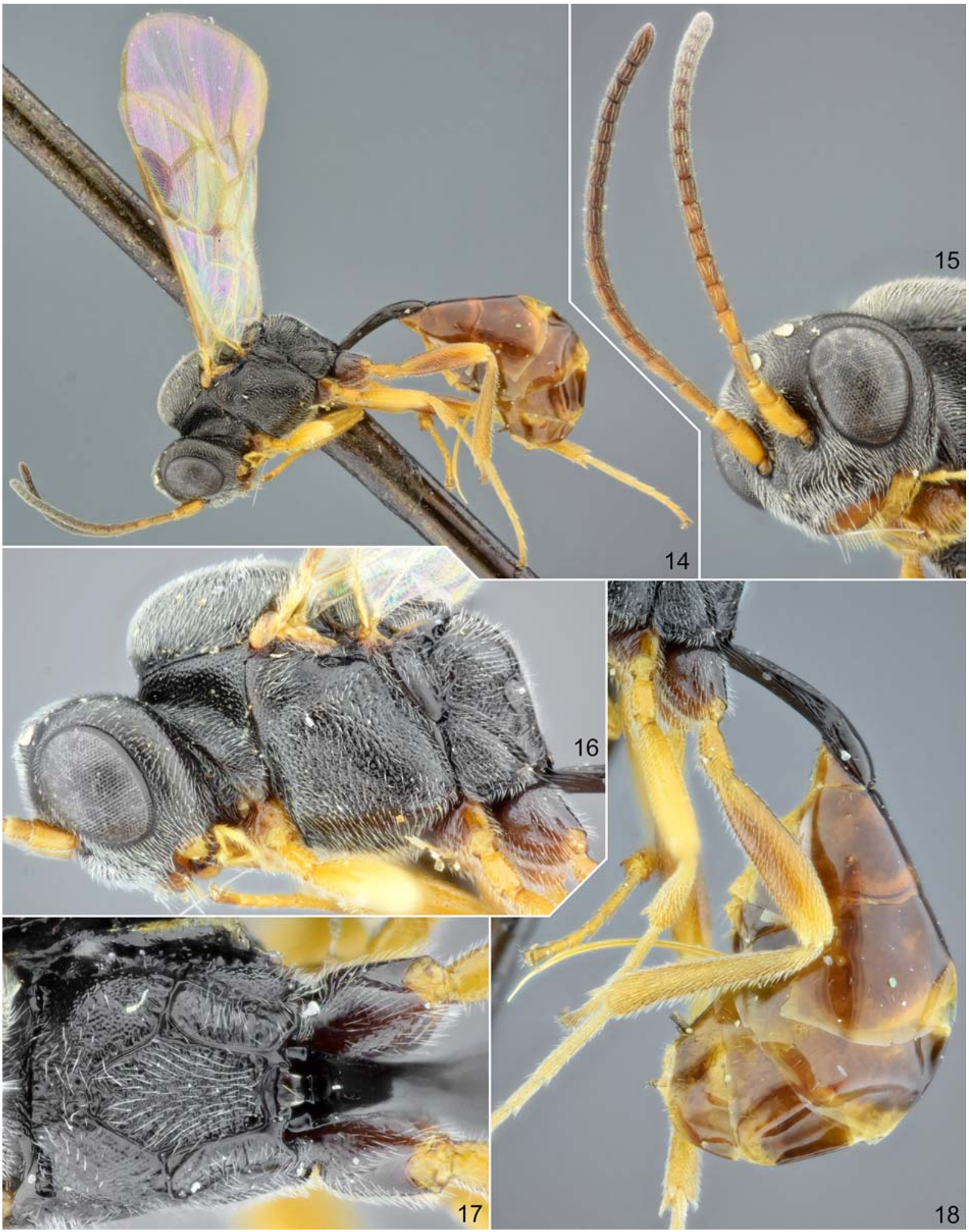

Figs 14-18. Phradis brevicauda, holotype female: 14 - habitus, lateral view; 15 - head with antennae, anterolateral view; 16 - head and mesosoma, lateral view; 17 - propodeum, dorsal view; 18 - metasoma with ovipositor, lateral view.

Рис. 14-18. Phradis brevicauda, голотип, самка: 14 - габитус, сбоку; 15 - голова с антеннами, спереди и сбоку; 16 - голова и мезосома, сбоку; 17 - проподеум, сверху; 18 - метасома с яйцекладом, сбоку. 
Ctenophion niger Horstmann, 2010: 100 Figs 2-8.

HOLOTYPE: + , USA, Colorado, Doolittle Ranch, Mt. Evans, 9800 ft. (= 2990 m), 1.VII.1964, coll. C. Dasch (AEIC). Li).

REFERENCES. Khalaim, Várkonyi, 2018: 166 (Finland:

MATERIAL EXAMINED. Canada, Alberta, Sunwapta Pass, Banff National Park, 7400 ft. (=2260 m), 1.VIII.1962, coll. W.R.M. Mason, 1 (paratype, ZSM).
DISTRIBUTION. Canada (Alberta, Yukon), USA (Alaska, Colorado), Finland (north).

Genus Phradis Förster, 1869

Type species: Thersilochus (Phradis) brevis Brischke, 1880.

DESCRIPTION. Small and medium sized species with body length usually between 2.5 and $4.0 \mathrm{~mm}$, thought largest species of the genus, e.g. P. gibbus (Holmgren, 1860) and P. longibasalis Khalaim, 2007, may have body length over $6.0 \mathrm{~mm}$.
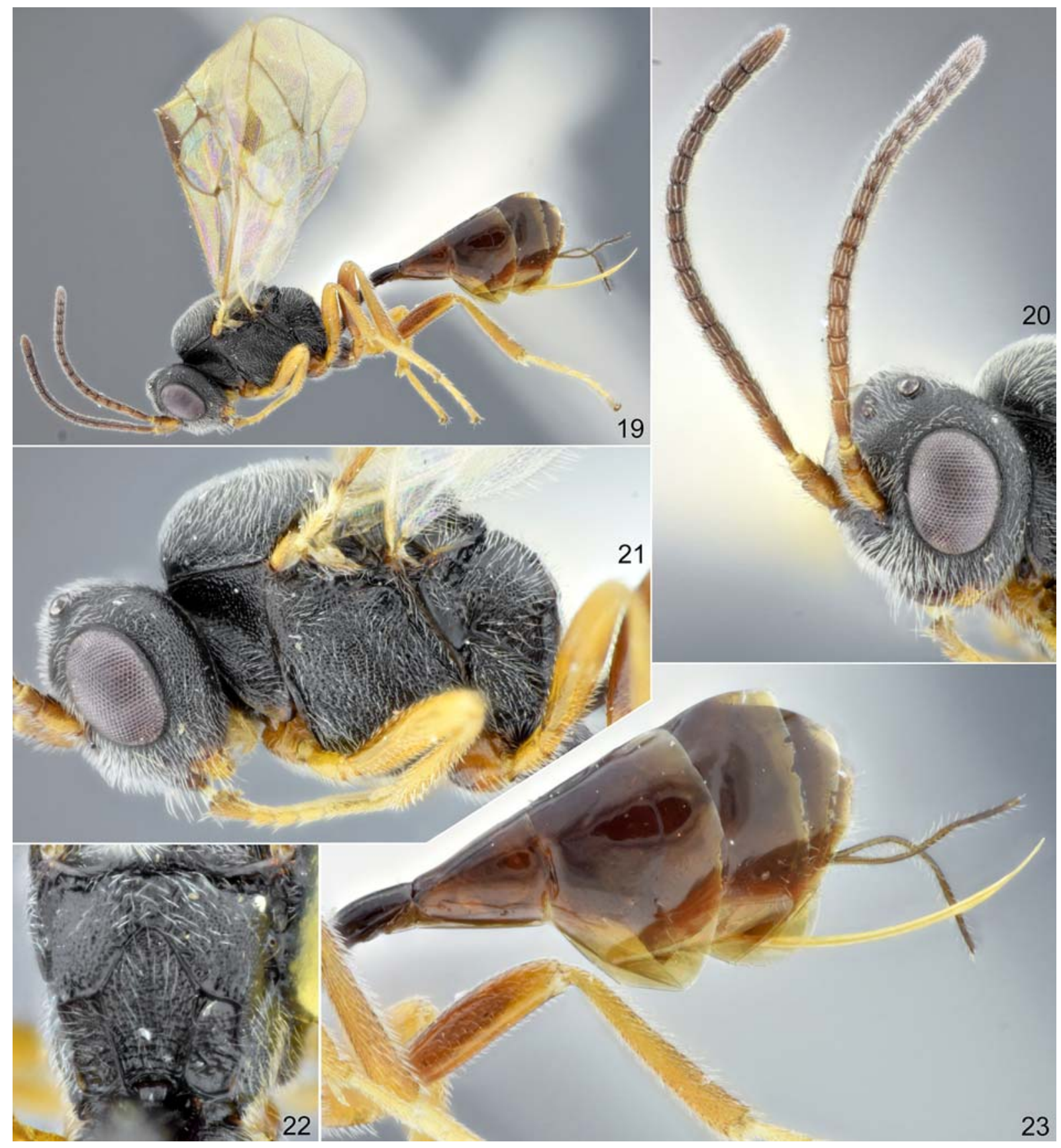

Figs 19-23. Phradis coriaceus, holotype female: 19 - habitus, lateral view; 20 - head with antennae, lateral view; 21 - head and mesosoma, lateral view; 22 - propodeum, dorsal view; 23 - apex of metasoma with ovipositor, lateral view.

Рис. 19-23. Phradis coriaceus, голотип, самка: 19 - габитус, сбоку; 20 - голова с антеннами, сбоку; 21 — голова и мезосома, сбоку; 22 - проподеум, сверху; 23 - вершина метасомы и яйцеклад, сбоку. 
Head, in dorsal view, usually with gena roundly tapered behind eyes, $0.5-1.1$ times as long as eye width. Antennal flagellum filiform or weakly clavate, sometimes very slender basally, with 8 to 24 flagellomeres; Nearctic P. curvicauda Horstmann, 2013 (Fig. 25) and P. flavipes Horstmann, 2013 (Fig. 33), and Palaearctic P. decameron Khalaim, 2004, possess flagellum with 7-9 flagellomeres, it is a minimum number of flagellomeres known in the family Ichneumonidae. Some flagellomeres bear more or less distinct finger-shaped structures on outer side near apex of each flagellomere [see Khalaim et al., 2009]. Mandible with upper tooth usually longer than lower tooth, but in several species (e.g. P. nigritulus (Gravenhorst, 1829), P. toreador Aubert, 1986) the lower tooth is longer. Clypeus lenticular,

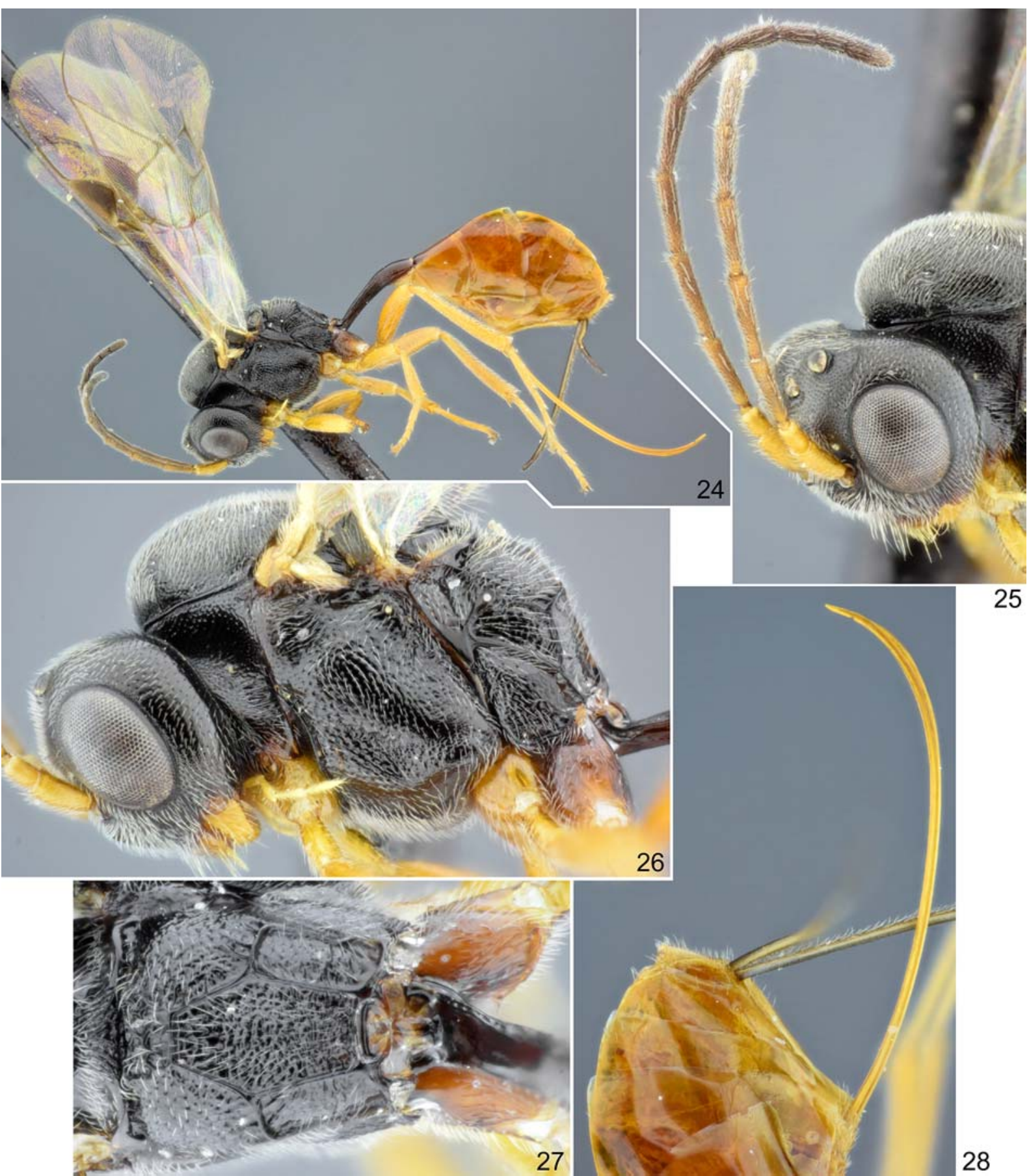

Figs 24-28. Phradis curvicauda, holotype female: 24 - habitus, lateral view; 25 - head with antennae, lateral view; 26 - head and mesosoma, lateral view; 27 - propodeum, dorsal view; 28 - apex of metasoma with ovipositor, lateral view.

Рис. 24-28. Phradis curvicauda, голотип, самка: 24 - габитус, сбоку; 25 — голова с антеннами, сбоку; 26 — голова и мезосома, сбоку; 27 - проподеум, сверху; 28 - вершина метасомы с яйцекладом, сбоку. 


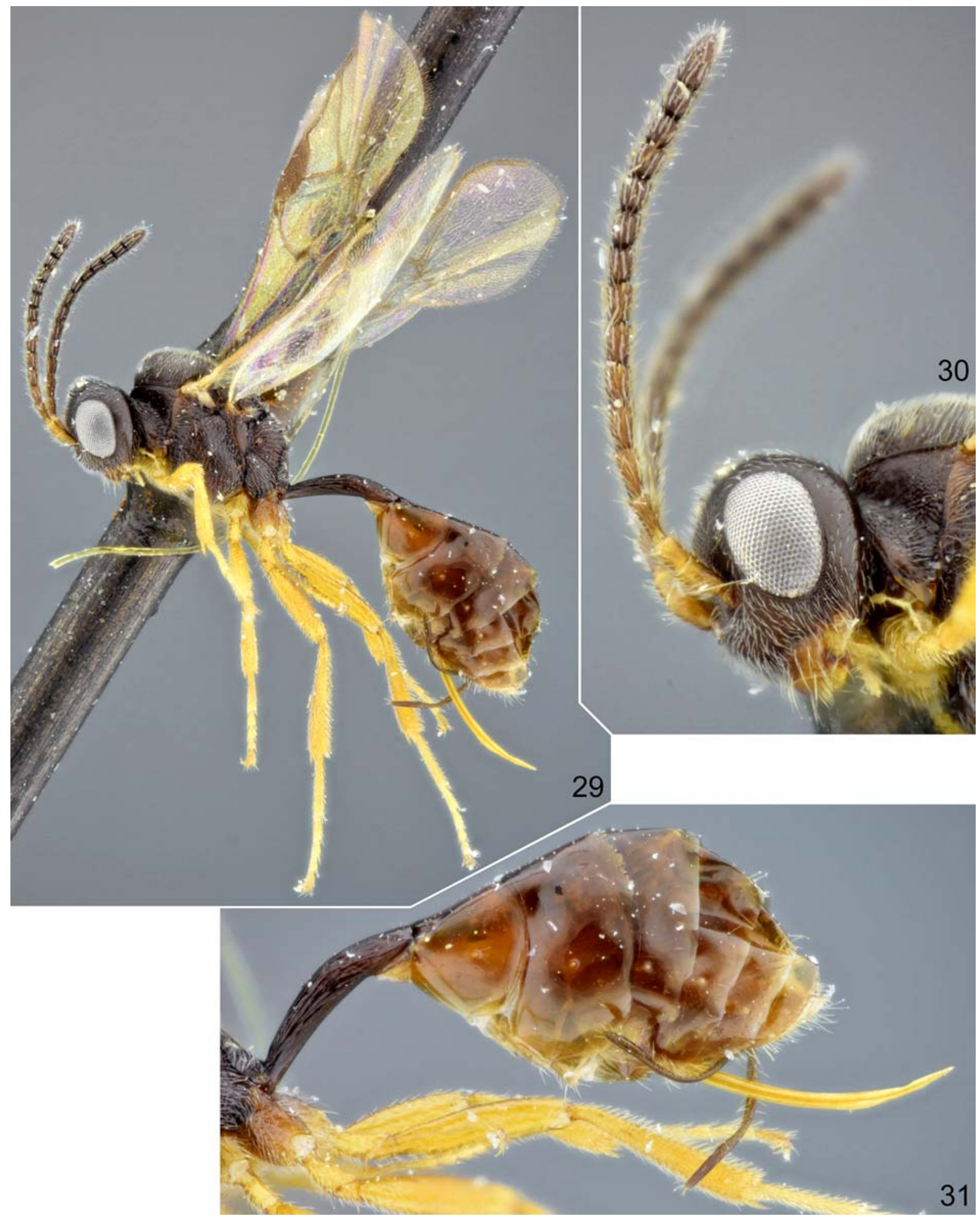

Figs 29-31. Phradis flavicoxa, holotype female: 29 - habitus, lateral view; 30 - head with antennae, lateral view; 31 - metasoma with ovipositor, lateral view.

Рис. 29-31. Phradis flavicoxa, голотип, самка: 29 — габитус, сбоку; 30 — голова с антеннами, сбоку; 31 - метасома с яйцекладом, сбоку. 
sometimes slightly truncated apically, with a transverse row of bristles near its lower margin. Hypostomal carina usually absent.

Notaulus usually with short wrinkle on anterolateral side of mesoscutum. Epicnemial carina with upper end bent forwards and reaching front margin of mesopleuron at level of lower $0.4-0.5$ of pronotum. Foveate groove of mesopleuron horizontal or weakly upcurved anteriorly, short and thin, or as weakly impressed area with transverse or irregular wrinkles, or completely absent. Propodeum usually with a pair of median longitudinal carinae bounding short basal area laterally (Figs 12, 17, 22, 27, etc.); basal area sometimes with irregular or longitudinal wrinkles; rarely basal area is long (in $P$. toreador) or substituted by furrow (in P. longibasalis). Propodeal spiracle round, separated from pleural carina by 2.0-6.0 times its own diameter.
Fore wing with second recurrent vein ( $2 \mathrm{~m}-\mathrm{cu})$ interstitial, rarely antefurcal or slightly postfurcal; in $P$. toreador vein $2 \mathrm{~m}$-cu completely absent. First and second sections of radius (Rs+2r and Rs) meeting at right or slightly acute angle. Second abscissa of postnervulus present, thus brachial cell is closed posteriorly. Hind wing with nervellus (cu1\&cu-a) subvertical to moderately reclivous.

Legs slender. Hind leg with tibia longer than femur and about twice as long as basitarsus. Longer hind tibial spur almost straight, with only distal end slightly curved. Tarsal claws not pectinate.

Metasoma behind tergite 1 more or less compressed laterally. First tergite slender, usually round in cross-section centrally, rarely slightly trapeziform or depressed. Glymma usually completely absent, rarely weak and isolated (i.e. not joining by furrow with ventral part of postpetiole); spiracle

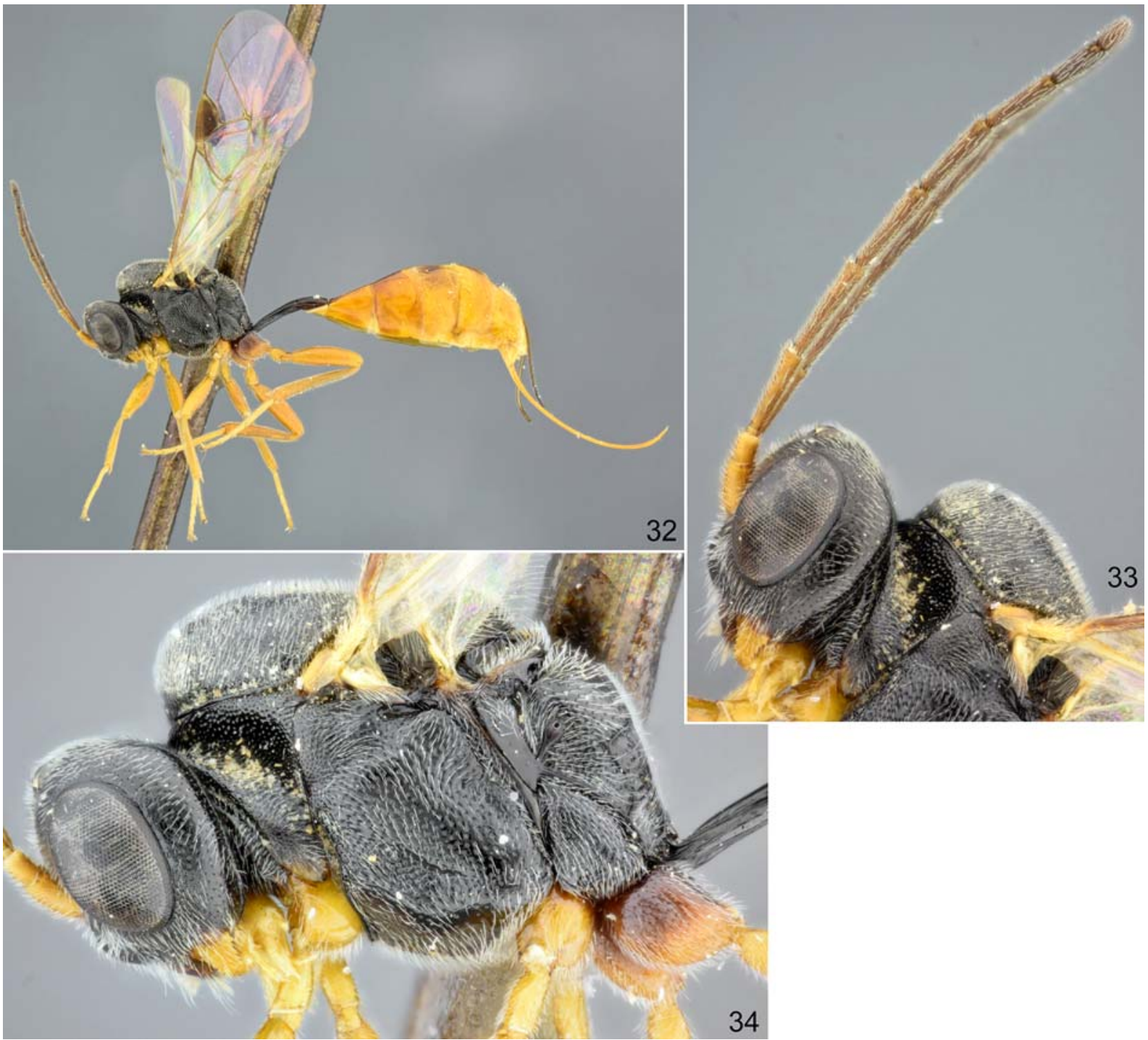

Figs 32-34. Phradis flavipes, holotype female: 32 - habitus, lateral view; 33 - head with antennae, lateral view; 34 - head and mesosoma, lateral view.

Рис. 32-34. Phradis flavipes, голотип, самка: 32 — габитус, сбоку; 33 — голова с антеннами, сбоку; 34 — голова и мезосома, сбоку. 
situated behind centre of first tergite; petiole not separated or weakly separated from postpetiole. Ovipositor slender, more or less upcurved, short to very long.

COMPOSITION. Moderately large genus with 63 known species worldwide: two species are known in the Afrotropical region [Khalaim, 2007], one in Australia [Khalaim, 2017], one in the Neotropical region [Khalaim, Bordera, 2012], 39 in the Palaearctic region [Khalaim, 2015; Yu et al., 2016] and 20 in the Nearctic region [Khalaim, 2002; Horstmann, 2013; Khalaim, Ruíz-Cancino, 2018].

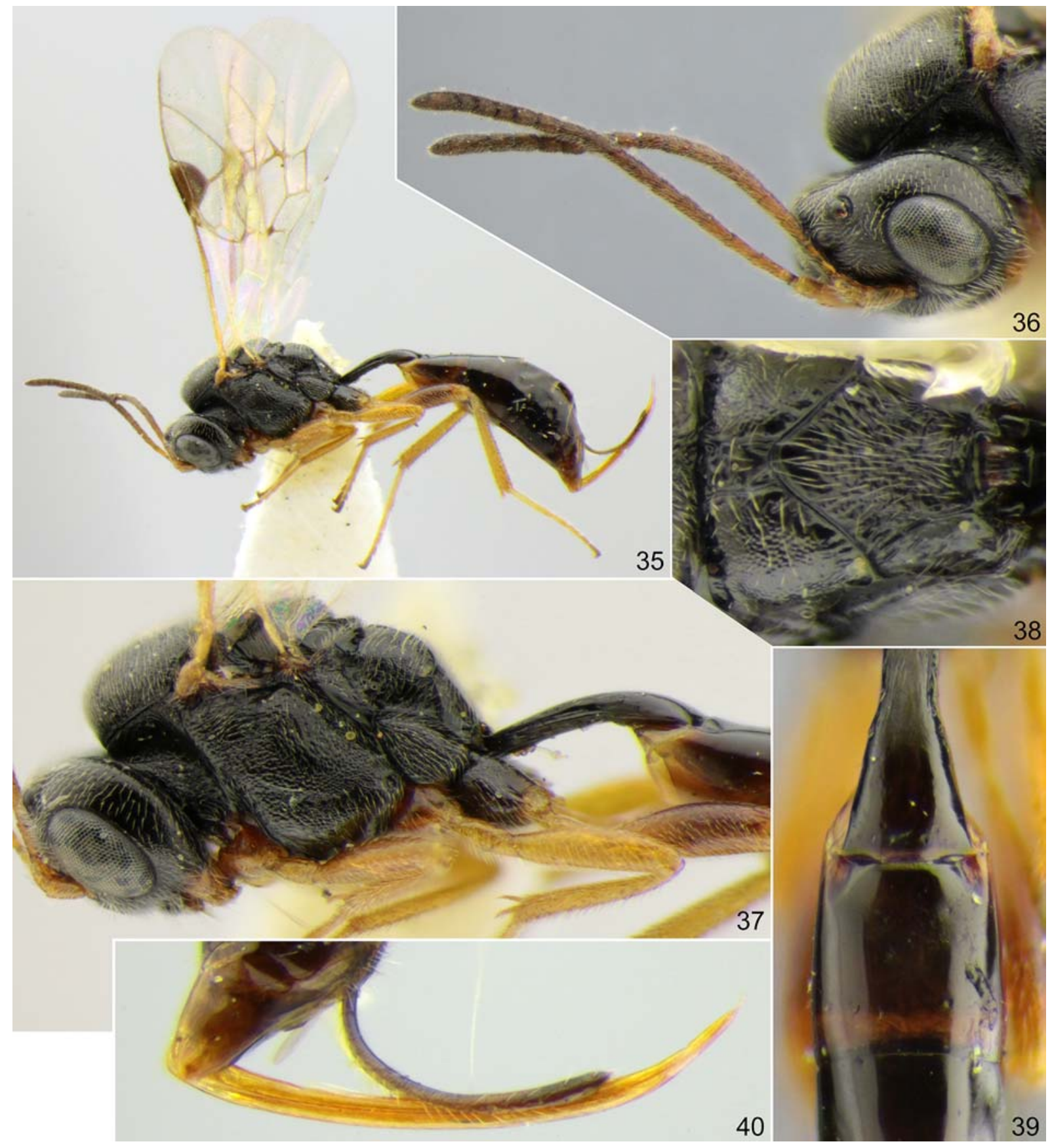

Figs 35-40. Phradis kasparyani, holotype female: 35 - habitus, lateral view; 36 - head with antennae, dorsolateral view; 37 - head, mesosoma and base of metasoma, lateral view; 38 - propodeum, dorsal view; 39 - apex of tergite 1 and tergite 2, dorsal view; 40 ovipositor, lateral view.

Рис. 35-40. Phradis kasparyani, голотип, самка: 35 - габитус, сбоку; 36 - голова с антеннами, сверху и сбоку; 37 — голова, мезосома и основание метасомы, сбоку; 38 - проподеум, сверху; 39 - вершина тергита 1 и тергит 2, сверху; 40 - яйцеклад, сбоку. 
1. Phradis basalis Horstmann, 2013: 70 Figs 9-13.

HOLOTYPE: + , USA, Michigan, Manistique, Jack pine [Pinus banksiana Lamb.], swept, 2.VI.1961, coll. W.R.M. Mason (CNC). DISTRIBUTION. USA (?Maryland, Michigan).

2. Phradis brevicauda Horstmann, 2013: 71 Figs 14-18.

HOLOTYPE: + , USA, California, Julian, 16.V.1974, coll. H. \& M. Townes (AEIC).
DISTRIBUTION. USA (California, ?Idaho, Wyoming).

\section{Phradis bufalosus Khalaim et Ruíz-Cancino,}

$$
\text { 2018: } 65
$$

HOLOTYPE: , Mexico, Tlaxcala, Nanacamilpa, Ejido Los Búfalos, N 19² ', W 98 $35^{\prime}$, bosque Pino-Encino, 2830-2900 m, Malaise trap, 4.IV-3.V.2016, coll. Y. Marquez \& A. Contreras (UNAM).

REMARKS. Photographs of this species were provided by Khalaim, Ruíz-Cancino [2018: 66, Figs 7-13].

DISTRIBUTION. Mexico (Tlaxcala).
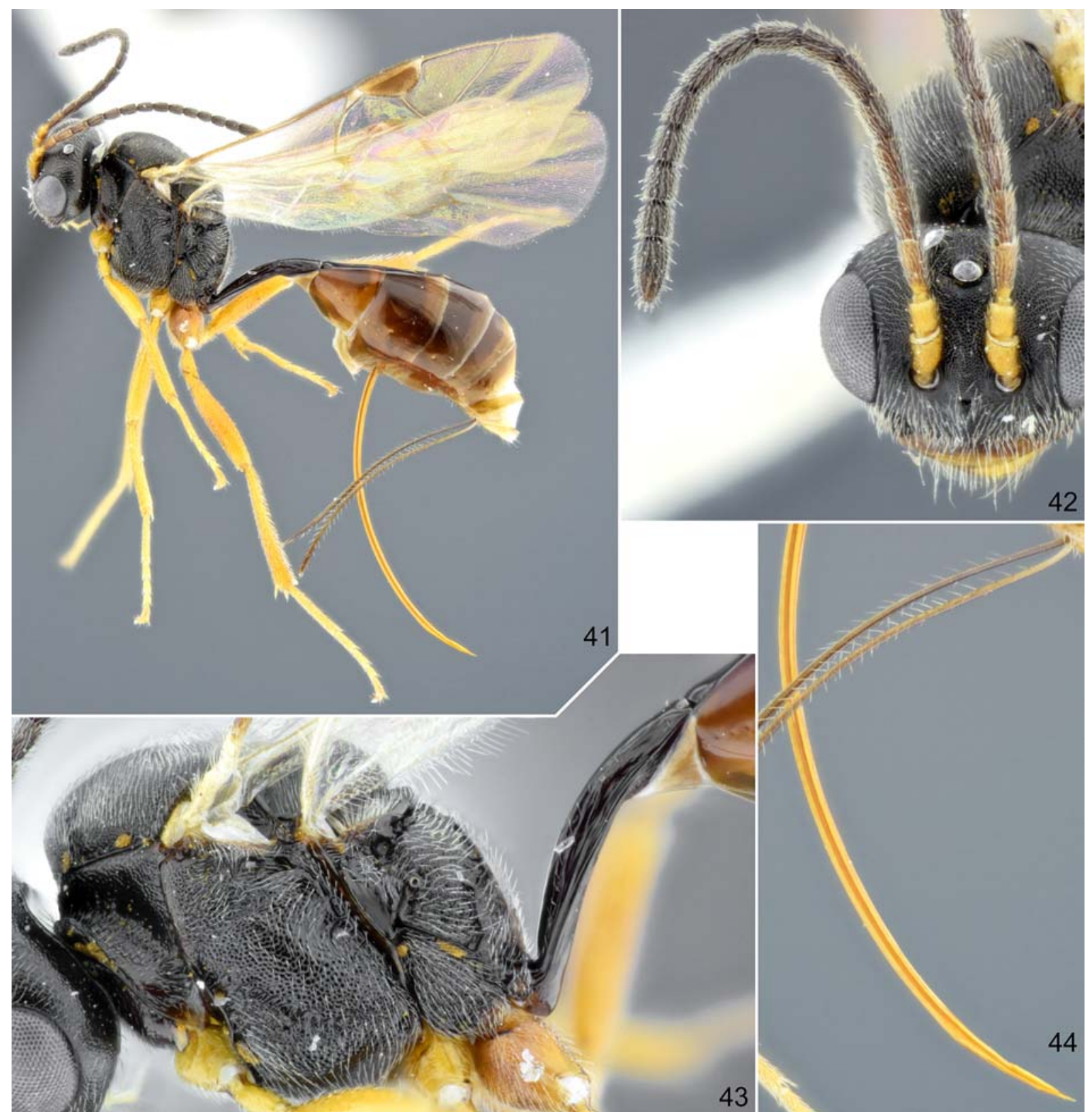

Figs 41-44. Phradis longicauda, holotype female: 41 - habitus, lateral view; 42 - head with antennae, front view; 43 - mesosoma and base of metasoma, lateral view; 44 - apex of ovipositor, lateral view.

Pис. 41-44. Phradis longicauda, голотип, самка: 41 - габитус, сбоку; 42 - голова с антеннами, спереди; 43 - мезосома и основание метасомы, сбоку; 44 - вершина яйцеклада, сбоку. 
4. Phradis coriaceus Horstmann, 2013: 72 Figs 19-23.

HOLOTYPE: + , USA, Maryland, Laurel, Malaise trap, 26.IV.1965 (CNC).

DISTRIBUTION. USA (Louisiana, Maryland).
5. Phradis curvicauda Horstmann, 2013: 73

Figs 24-28.

HOLOTYPE: + , USA, Oregon, Hyatt Reservoir, 20.VI.1978, coll. H. \& M. Townes (AEIC).

DISTRIBUTION. USA (Oregon, Wyoming).
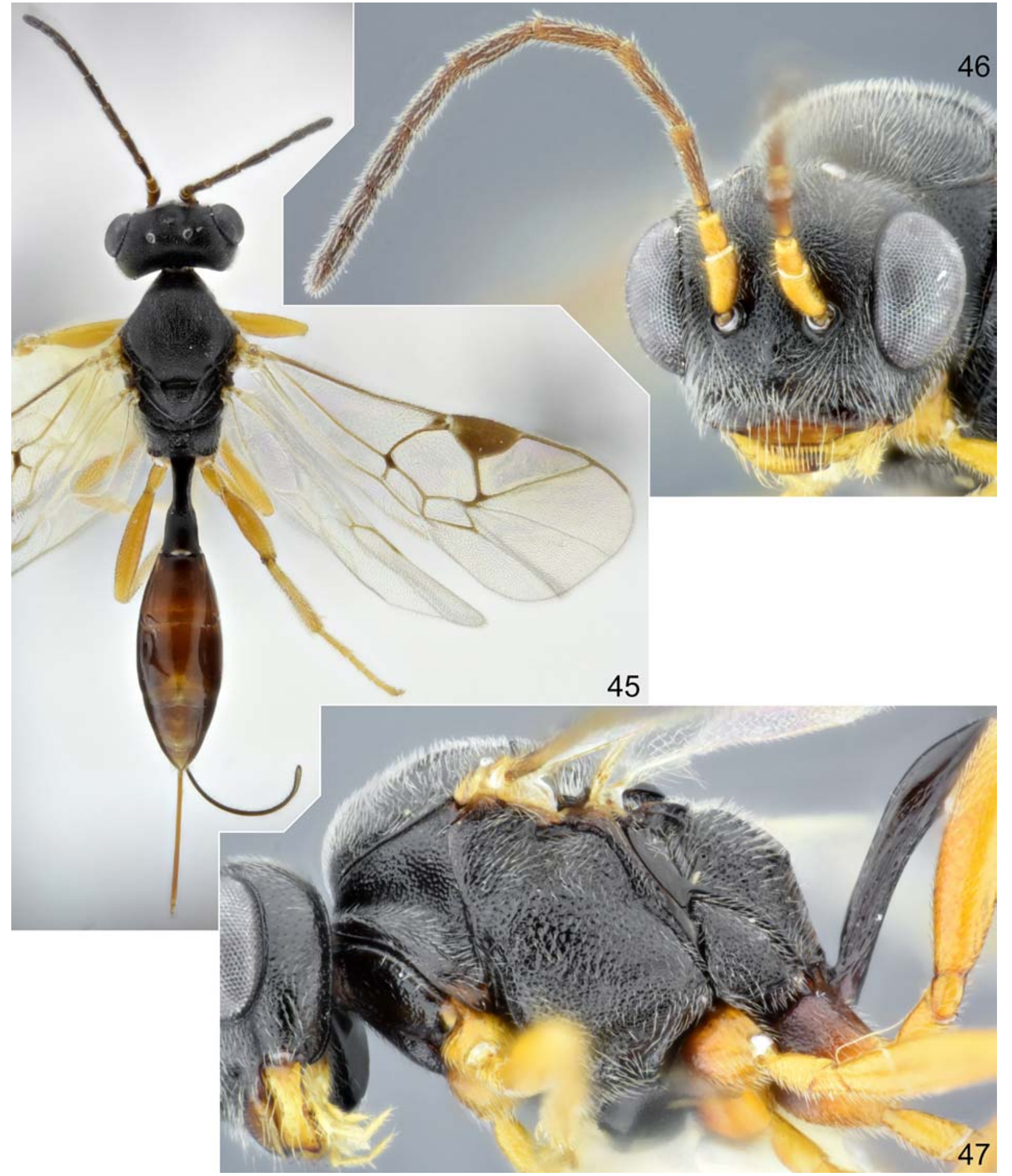

Figs 45-47. Phradis minor, holotype female: 45 - habitus, dorsal view; 46 - head with antenna, front view; 47 - mesosoma and base of metasoma, lateral view.

Рис. 45-47. Phradis minor, голотип, самка: 45 — габитус, сбоку; 46 — голова с антенной, спереди; 47 - мезосома и основание метасомы, сбоку. 


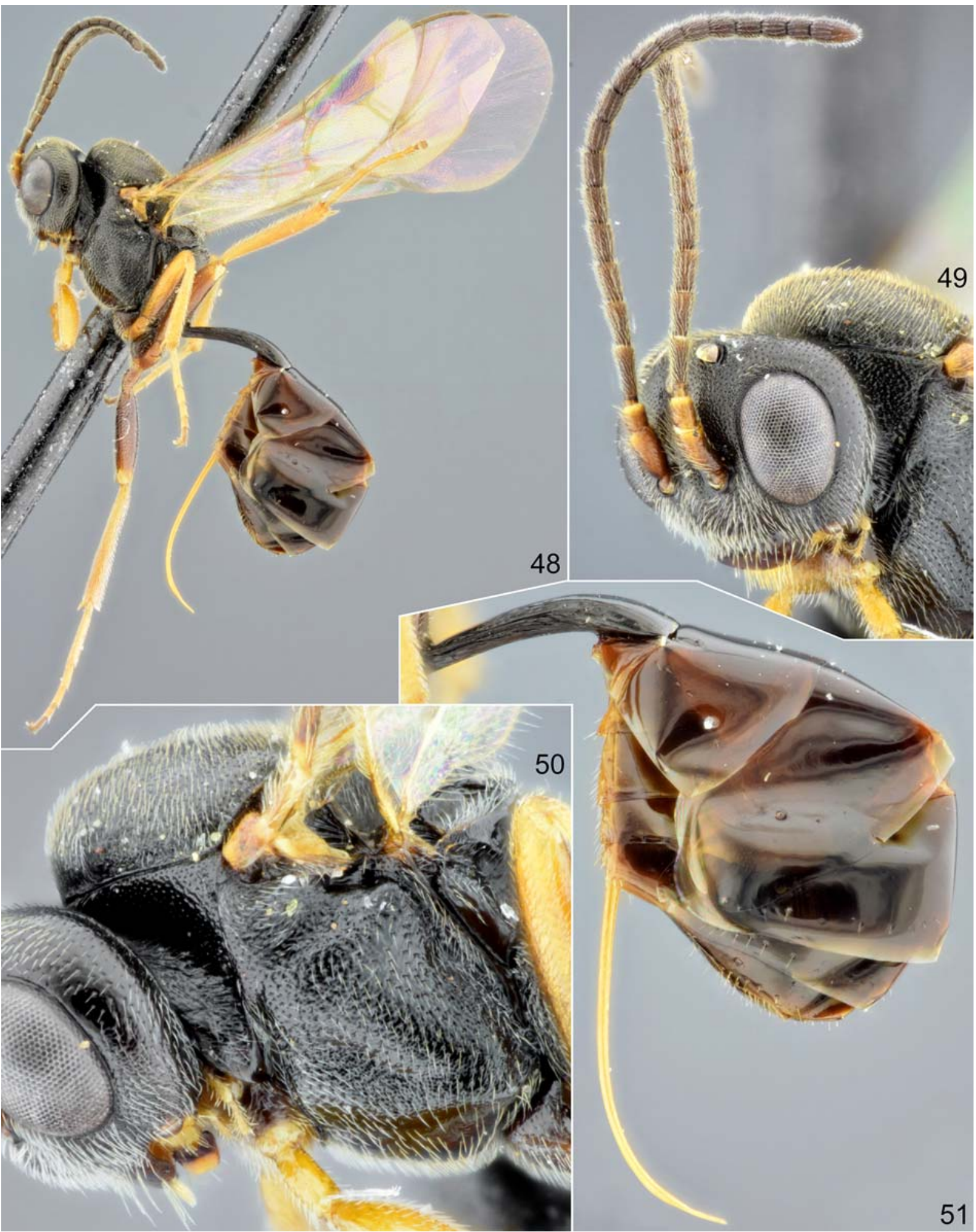

Figs 48-51. Phradis nigricoxa, holotype female: 48 - habitus, lateral view; 49 - head with antennae, anterolateral view; 50 - head and mesosoma, lateral view; 51 - metasoma with ovipositor, lateral view.

Рис. 48-51. Phradis nigricoxa, голотип, самка: 48 — габитус, сбоку; 49 — голова с антеннами, спереди и сбоку; 50 — голова и мезосома, сбоку; 51 - метасома с яйцекладом, сбоку. 


\section{Phradis flavicoxa Horstmann, 2013: 75} Figs 29-31.

HOLOTYPE: 9 , Canada, Newfoundland and Labrador, South Branch, Malaise trap, VII.1974, coll. Heinrich (CNC).

DISTRIBUTION. Canada (British Columbia, Manitoba, Newfoundland and Labrador, Ontario, Quebec), USA (California, Iowa, Washington).
7. Phradis flavipes Horstmann, 2013: 76

Figs 32-34.

HOLOTYPE: + , USA, California, Julian, 24.V.1974, coll. H. \& M. Townes (AEIC) DISTRIBUTION. USA (California).

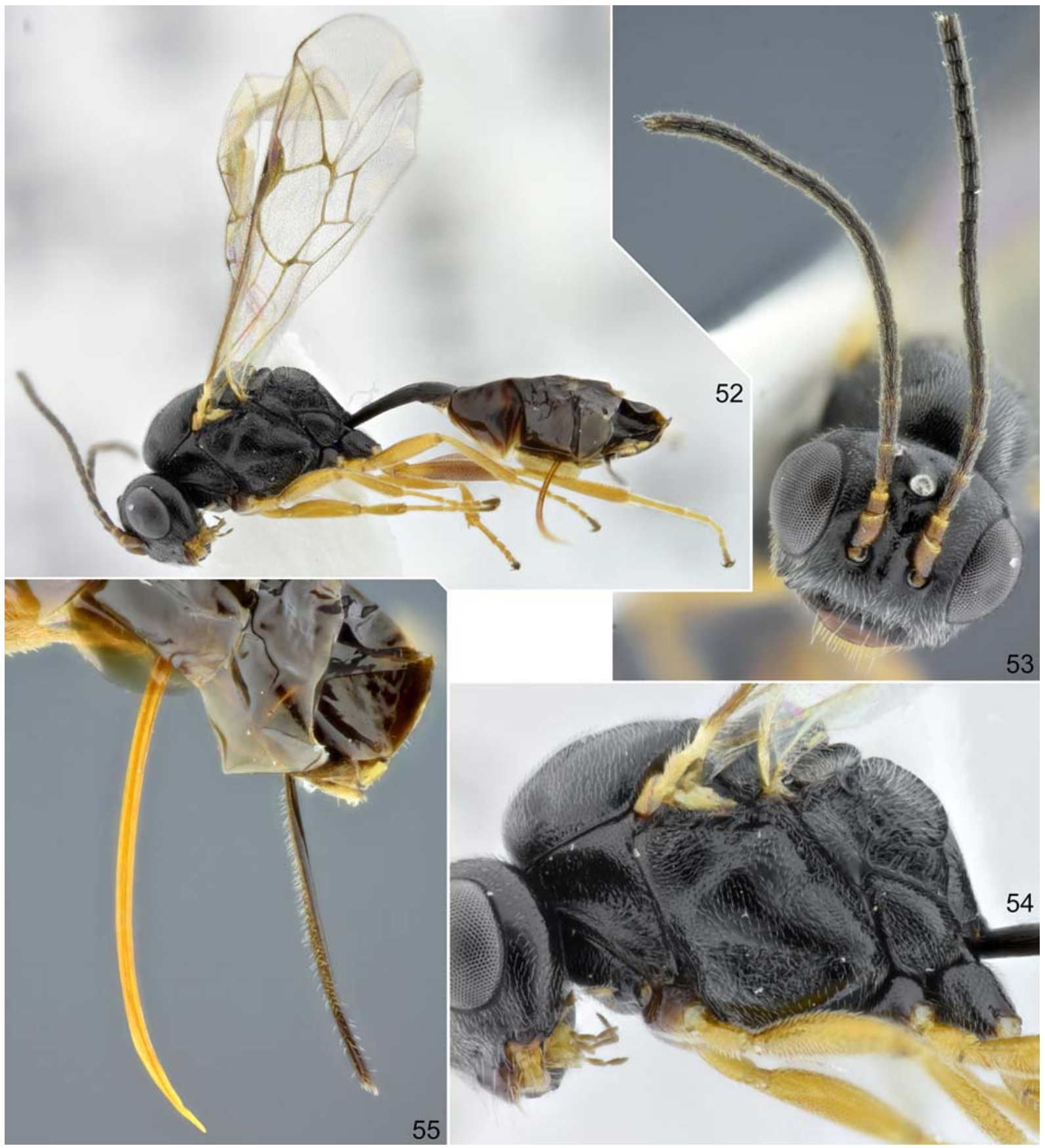

Figs 52-55. Phradis nitidipleuris, holotype female: 52 - habitus, lateral view; 53 - head with antennae, front view; 54 - mesosoma, lateral view; 55 - apex of metasoma with ovipositor, lateral view.

Рис. 52-55. Phradis nitidipleuris, голотип, самка: 52 — габитус, сбоку; 53 — голова с антеннами, спереди; 54 — мезосома, сбоку; 55 - вершина метасомы с яйцекладом, сбоку. 
8. Phradis kasparyani Khalaim, 2002: 221 Figs 35-40.

HOLOTYPE: + , USA, California, San-Bernardino, $1600 \mathrm{~m}$, Milk Creek, Quercus, 10.V.1988, coll. D.R. Kasparyan (ZIN).

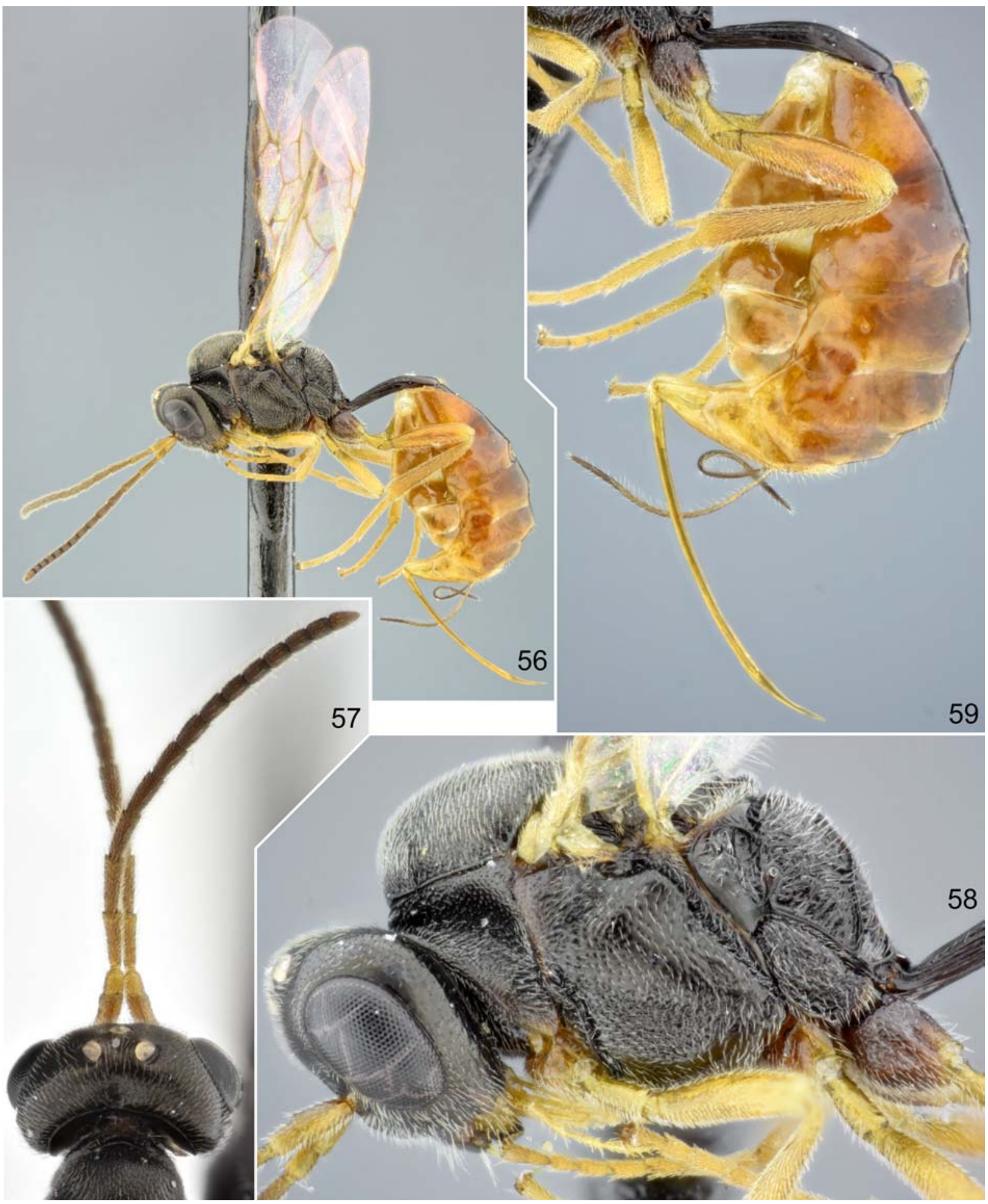

Figs 56-59. Phradis nitidus, holotype female: 56 - habitus, lateral view; 57 - head with antennae, dorsal view; 58 - head and mesosoma, lateral view; 59 - metasoma with ovipositor, lateral view.

Рис. 56-59. Phradis nitidus, голотип, самка: 56 - габитус, сбоку; 57 - голова с антеннами, сверху; 58 — голова и мезосома, сбоку; 59 - метасома с яйцекладом, сбоку.
REFERENCES, Horstmann, 2013: 77 (USA: Arizona, California, Oregon).DISTRIBUTION. USA (Arizona, California, Oregon) 
9. Phradis longicauda Horstmann, 2013: 78

Figs 41-44.

HOLOTYPE: + , USA, Texas, Brazos Co., Lick Creek Park, $2-$ 9.II.2008, coll. R. Wharton (TAMU).

DISTRIBUTION. USA (Texas).

10. Phradis minor Horstmann, 2013: 79

Figs 45-47.

HOLOTYPE: + , USA, Texas, Walker Co., $10 \mathrm{mi} . \mathrm{W}$ of New Waverly, 24.III.1991, coll. R. Wharton (TAMU).

DISTRIBUTION. USA (Louisiana, Texas).
11. Phradis nanacamilpus

Khalaim et Ruíz-Cancino,

$$
\text { 2018: } 68
$$

HOLOTYPE: + , Mexico, Tlaxcala, Nanacamilpa, Ejido Los Búfalos, N 19²8', W 98³5', bosque Pino-Encino, 2830-2900 m, Malaise trap, 3-30.V.2016, coll. Y. Marquez \& A. Contreras (UNAM).

REMARKS. Photographs of this species were provided by Khalaim, Ruíz-Cancino [2008: 69, Figs 14-21].

DISTRIBUTION. Mexico (Tlaxcala).

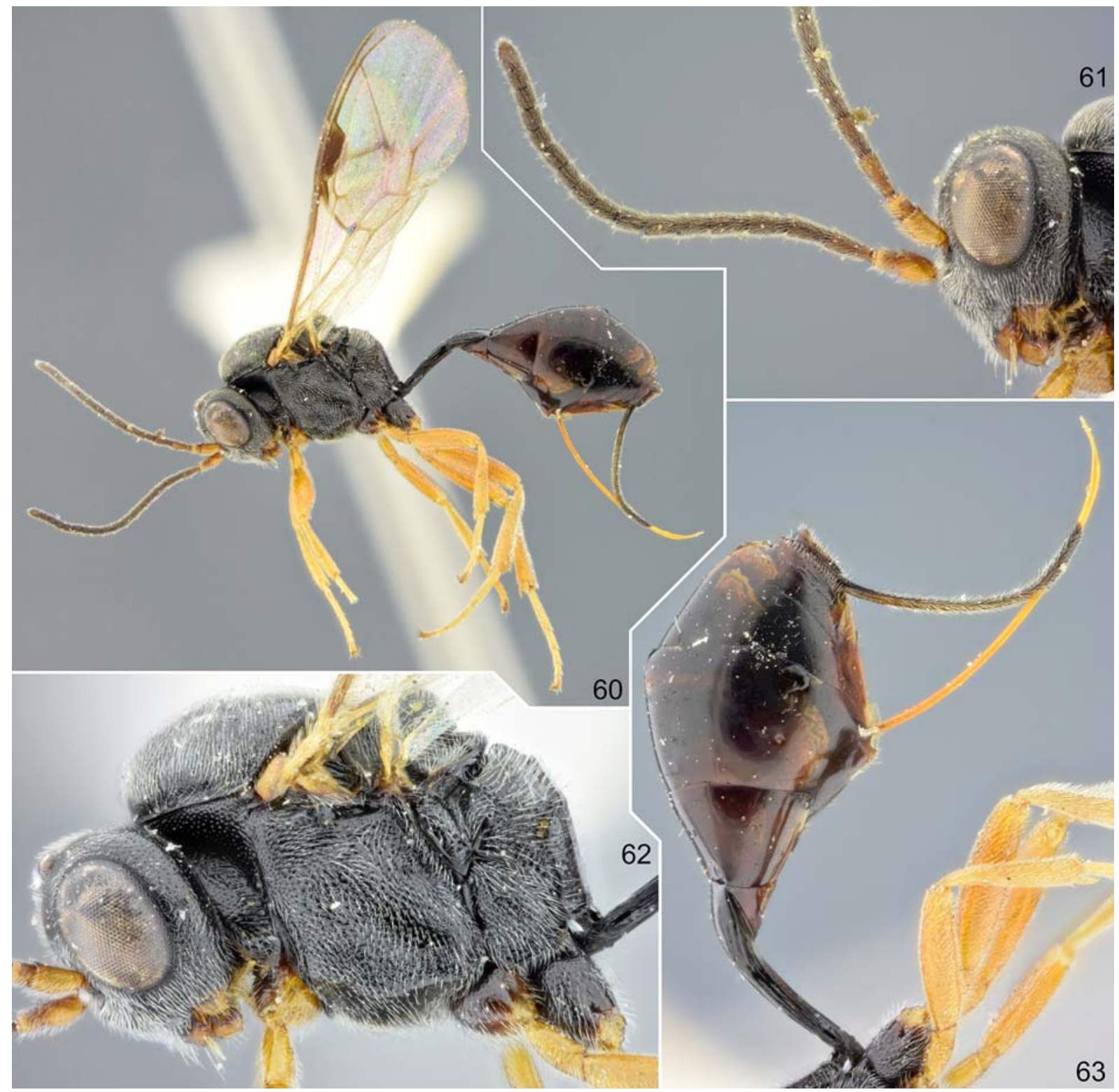

Figs 60-63. Phradis punctatus, holotype female: 60 - habitus, lateral view; 61 - head with antennae, lateral view; 62 - head and mesosoma, lateral view; 63 - metasoma with ovipositor, lateral view.

Рис. 60-63. Phradis punctatus, голотип, самка: 60 — габитус, сбоку; 61 — голова с антеннами, сбоку; 62 — голова и мезосома, соку; 63 - метасома с яйцекладом, сбоку. 
12. Phradis nigricoxa Horstmann, 2013: 80 Figs 48-51.

HOLOTYPE: 9 , USA, Oregon, Hyatt Reservoir, 29.VI.1978, coll. H. \& M. Townes (AEIC)

DISTRIBUTION. USA (?Idaho, Oregon).

13. Phradis nitidipleuris Horstmann, 2013: 82 Figs 52-55.

HOLOTYPE: + , USA, Wyoming, Albany Co., Medicine Bow National Forest, Yellow Pines Camp Area near Pole Creek, Malaise trap near willows, 4-11.VI.2003, coll. S.R. Shaw (ESUW).

DISTRIBUTION. USA (Wyoming).
14. Phradis nitidus Horstmann, 2013: 83

Figs 56-59.

HOLOTYPE: + , Canada, Yukon, Haines Junction, Dezadeash Lake, 700-900 m, 2.VIII.1988, coll. S. \& J. Peck (AEIC).

DISTRIBUTION. Canada (Alberta, British Columbia, Yukon), USA (?Arizona, Colorado, Idaho, New Mexico, ?Washington, Wyoming).

\section{Phradis punctatus Horstmann, 2013: 84} Figs 60-63.

HOLOTYPE: + , Canada, Quebec, Kazabazua, taken on pines, 6.VI.1956, coll. W.R.M. Mason (CNC).

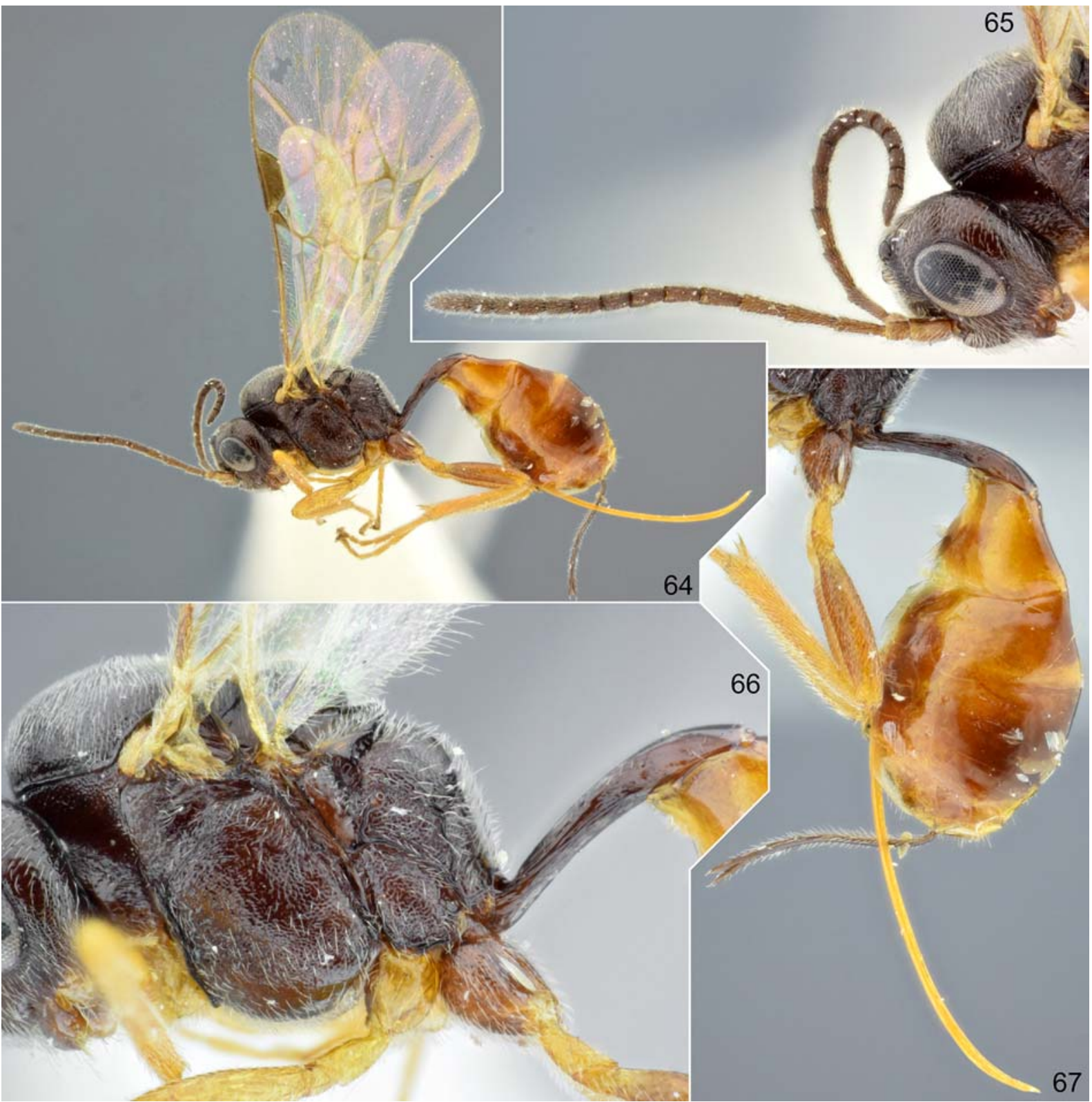

Figs 64-67. Phradis rarus, holotype female: 64 - habitus, lateral view; 65 - head with antennae, lateral view; 66 - mesosoma and base of metasoma, lateral view; 67 - metasoma with ovipositor, lateral view.

Рис. 64-67. Phradis rarus, голотип, самка: 64 - габитус, сбоку; 65 - голова с антеннами, сбоку; 66 - мезосома и основание метасомы, сбоку; 67 - метасома с яйцекладом, сбоку. 
DISTRIBUTION. Canada (Quebec).

16. Phradis rarus Horstmann, 2013: 85

Figs 64-67.

HOLOTYPE: 9 Canada, Alberta, Waterton, 18.VI.1957, coll. O. Peck (CNC).

DISTRIBUTION. Canada (Alberta).

17. Phradis striatus Horstmann, 2013: 86

Figs 68-71.

HOLOTYPE: ㅇ, Canada, Quebec, Kazabazua, taken on pines, 6.VI.1956, coll. W.R.M. Mason (CNC).

DISTRIBUTION. Canada (Quebec), USA (?Maryland, ?Virginia).
18. Phradis sulcator Horstmann, 2013: 88

Figs 72-75.

HOLOTYPE: 9 , USA, Illinois, Florence, 22.V.1941, coll. H.K. Townes [erroneously "K.H. Townes" in the original description] (AEIC).

DISTRIBUTION. Canada (Ontario), USA (Illinois, Indiana, Iowa, Maryland, Massachusetts, Michigan, Missouri, New York, Ohio, Pennsylvania, South Carolina, Virginia, Wisconsin).

19. Phradis tenuicauda Horstmann, 2013: 89

Figs 76-78.

HOLOTYPE:, , USA, Idaho, Idaho City, 9.VI.1978, coll. H. \& M. Townes (AEIC).

DISTRIBUTION. USA (Idaho).

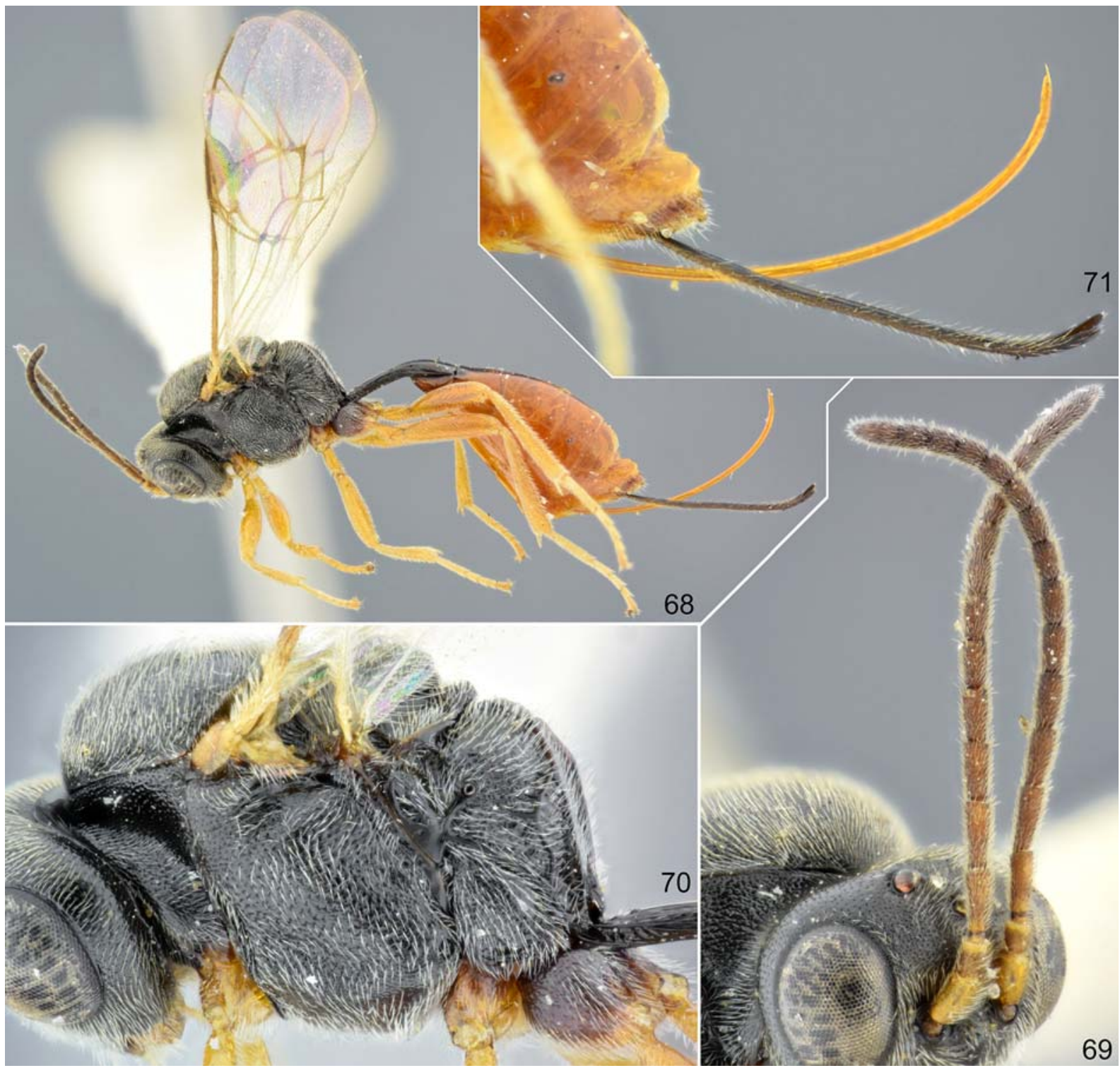

Figs 68-71. Phradis striatus, holotype female: 68 - habitus, lateral view; 69 - head with antennae, anterolateral view; 70 - mesosoma, lateral view; 71 - ovipositor, lateral view.

Рис. 68-71. Phradis striatus, голотип, самка: 68 - габитус, сбоку; 69 - голова с антеннами, спереди и сбоку; 70 — мезосома, сбоку; 71 - яйцеклад, сбоку. 
20. Phradis texanus Horstmann, 2013: 90

Figs 79-84.

HOLOTYPE: + , USA, Texas, Bastrop Co., Bastrop State Park, Malaise trap, 12-17.IV.1991, coll. Wharton \& Geiselbrecht (TAMU) DISTRIBUTION. USA (Texas).

Acknowledgements. I am thankful to Stefan and Olga Schmidt for their organizing my staying in the ZSM collection in May 2016, and Bruno Cancian de Araujo for his help with taking colour photographs in the ZSM. I also thank Martin Schwarz (Kirchschlag, Austria) and Andrei E. Humala (Forest Research Institute of the Russian Academy of Sciences, Petrozavodsk, Russia) for reviewing the paper.
This work was supported by the PRODEP project "Taxonomical and biological studies of pests and natural enemies in Mexico" and the grant no. 19-04-00027 from the Russian Foundation for Basic Research, and performed in the framework of the Russian State Research Project no. 01201351189.

\section{References}

Horstmann K. 2010. Revisions of Nearctic Tersilochinae II. Genera Allophrys Förster, Barycnemis Förster, Ctenophion gen. nov., Sathropterus Förster, Spinolochus Horstmann and Stethantyx Townes (Hymenoptera, Ichneumonidae) // Spixiana. Bd.33. H.1. P.73-109.

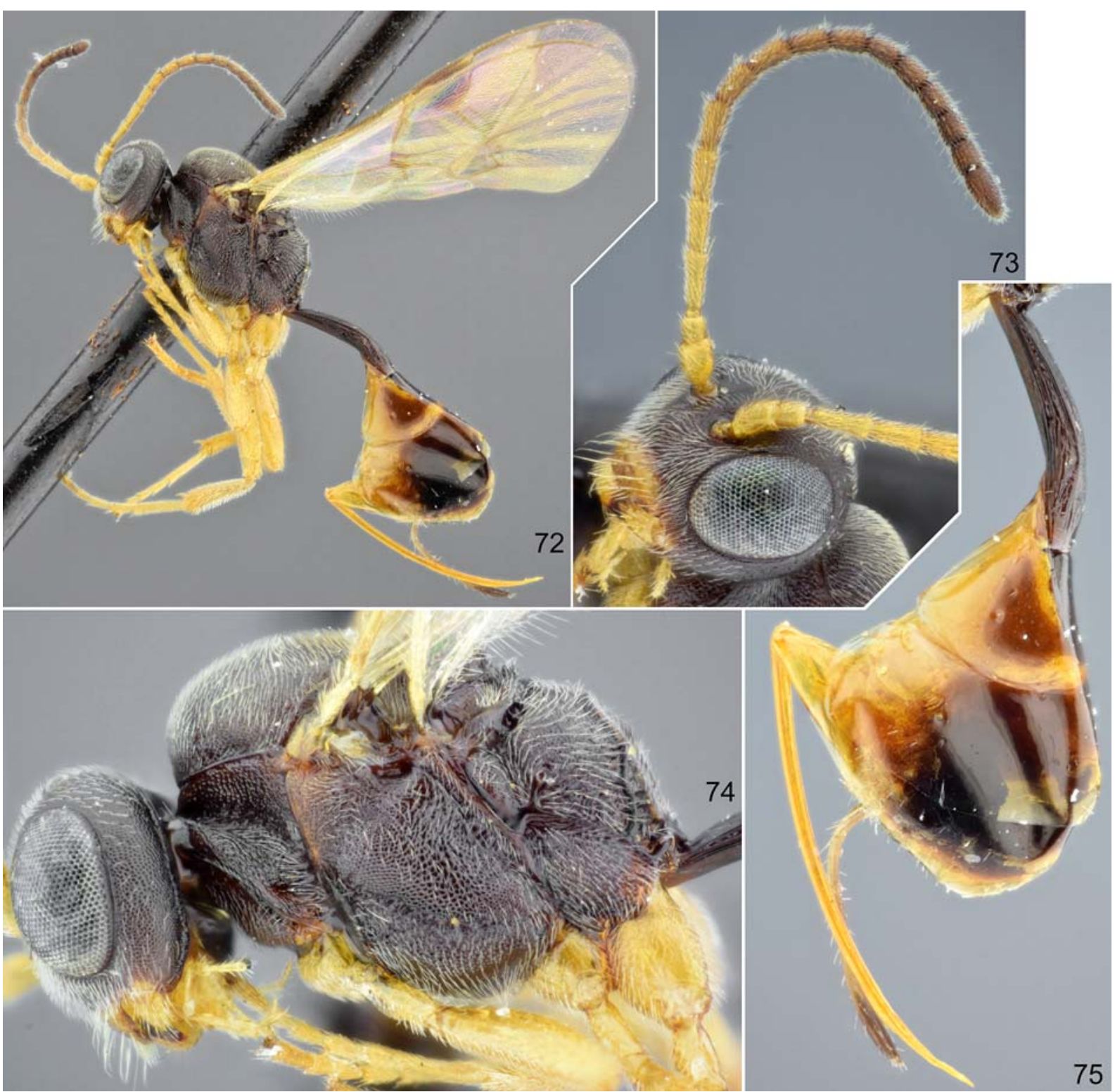

Figs 72-75. Phradis sulcator, holotype female: 72 - habitus, lateral view; 73 - head with antenna, anterolateral view; 74 - head and mesosoma, lateral view; 75 - metasoma with ovipositor, lateral view.

Рис. 72-75. Phradis sulcator, голотип, самка: 72 - габитус, сбоку; 73 - голова с антенной, спереди и сбоку; 74 - голова и мезосома, сбоку; 75 - метасома с яйцекладом, сбоку. 
Horstmann K. 2013. Revisions of Nearctic Tersilochinae IV. Genus Phradis Förster (Hymenoptera, Ichneumonidae) // Spixiana. Bd.36. H.1. P.67-92.

Khalaim A.I. 2002. A new species of the genus Phradis Förster, 1869 from the USA (Hymenoptera: Ichneumonidae: Tersilochinae) // Russian Entomological Journal. Vol.11. No.2. P.221-222.

Khalaim A.I. 2007. First records of Meggoleus, Heterocola and Phradis (Hymenoptera: Ichneumonidae: Tersilochinae) from the Afrotropical region, with description of four new species // African Invertebrates. Vol.48. No.2. P.101-110.

Khalaim A.I. 2015. A new species of Phradis Förster (Hymenoptera: Ichneumonidae: Tersilochinae) from Japan // Zoosystematica Rossica. Vol.24. No.1. P.108-112.
Khalaim A.I. 2017. New species of the genera Australochus Khalaim and Phradis Förster (Hymenoptera: Ichneumonidae: Tersilochinae) from Australia // Russian Entomological Journal. Vol.26. No.4. P.327-331.

Khalaim A.I., Bordera S. 2012. First record of the genus Phradis Förster (Hymenoptera, Ichneumonidae, Tersilochinae) from the Neotropical Region // ZooKeys. Vol.169. P.31-38.

Khalaim A.I., Bordera S., Rodríguez-Berrío A. 2009. A review of the European species of Phradis (Hymenoptera: Ichneumonidae: Tersilochinae), with description of a new species from Spain // European Journal of Entomology. Vol.106. No.1. P.107-118.

Khalaim A.I, Ruíz-Cancino E 2018. First record of the genera Diaparsis Förster and Phradis Förster (Hymenoptera, Ichneu-

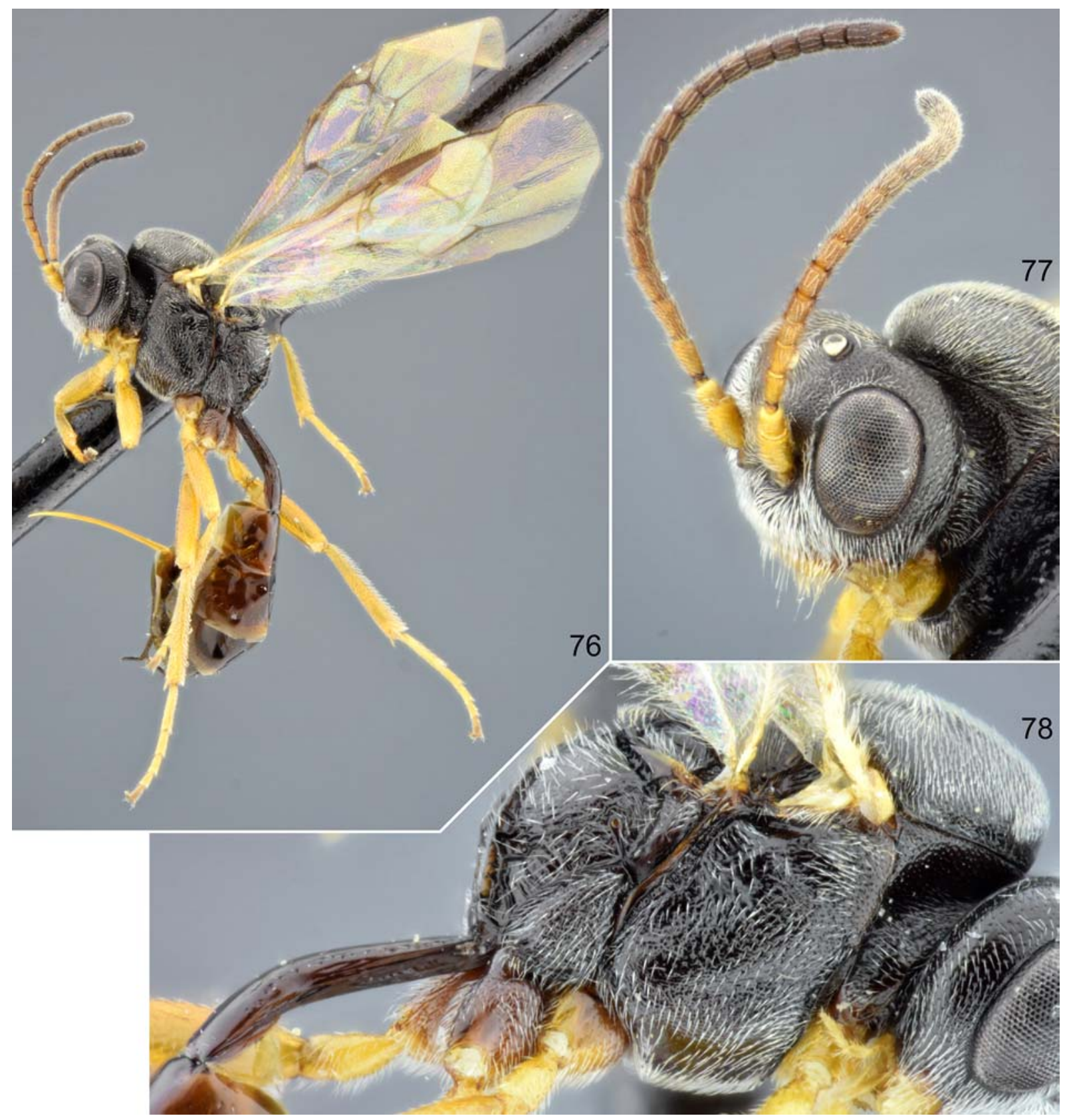

Figs 76-78. Phradis tenuicauda, holotype female: 76 - habitus, lateral view; 77 - head with antennae, anterolateral view; 78 mesosoma and base of metasoma, lateral view.

Рис. 76-78. Phradis tenuicauda, голотип, самка: 76 — габитус, сбоку; 77 — голова с антеннами, спереди и сбоку; 78 — мезосома и основание метасомы, сбоку. 
monidae, Tersilochinae) from Mexico // Journal of Hymenoptera Research. Vol.63. P.61-72.

Khalaim A.I., Várkonyi G. 2018. A review of Tersilochinae (Hymenoptera: Ichneumonidae) of Finland. Part 1: taxonomy //
Zootaxa. Vol.4369. No.2. P.151-185.

Yu D.S.K., van Achterberg C., Horstmann K. 2016. Taxapad 2016, Ichneumonoidea 2015. Database on flash-drive. Nepean, Ontario, Canada.

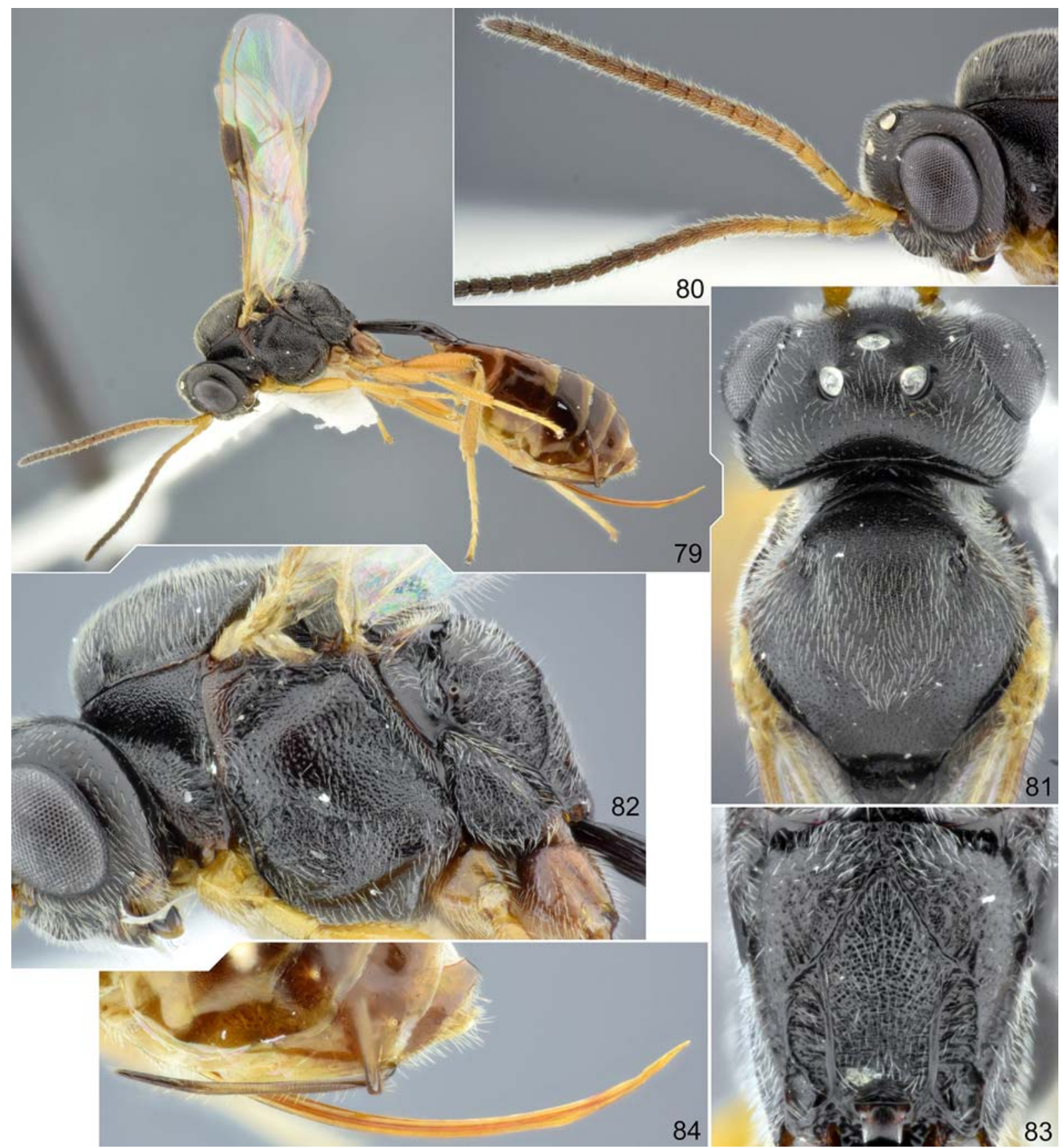

Figs 79-84. Phradis texanus, holotype female: 79 - habitus, lateral view; 80 - head with antennae, lateral view; 81 - head and mesoscutum, dorsal view; 82 - mesosoma, lateral view; 83 - propodeum, dorsal view; 84 - ovipositor, lateral view.

Рис. 79-84. Phradis texanus, голотип, самка: 79 - габитус, сбоку; 80 - голова с антеннами, сбоку; 81 — голова и мезоскутум, сверху; 82 - мезосома, сбоку; 83 - проподеум, сверху; 84 - яйцеклад, сбоку. 